\title{
Nehmen wir den «grauen Tsunami» ernst genug?
}

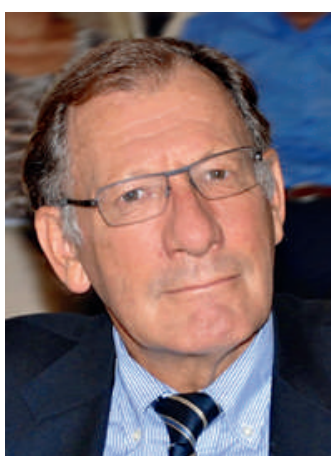

Jean Martin
Die «grauen Panther» sind bekannt - eine Bewegung, die in den USA in den frühen 1970er Jahren ihren Anfang nahm, in der Senioren sich politisch für die Belange älterer Menschen einsetzten. Heute spricht man vom «grauen Tsunami» und meint damit die Herausforderungen, vor die das Altern der Bevölkerung die gesamte Gesellschaft (ihr medizinisch-soziales System, das Rentensystem usw.) stellt. Die öffentliche Hand muss ihre Planungen darauf einstellen, doch erhalten die Zahlen zum Thema wirklich die Beachtung, die ihnen gebührt? (Ich erinnere daran, dass ausser bei starker Immigration die Bevölkerungsprognosen für ein Land auf viele Jahre hinaus zuverlässig sind.) Bei der Regierung des Kantons Waadt ist dies offensichtlich so, denn hier wurde der schwierige und exponierte Posten an der Spitze des Amts für Gesundheit mit einer Fachärztin für Geriatrie besetzt. Zitat Dr. Stefanie Monod: «Wenn wir unser Gesundheitssystem nicht hinterfragen, erleiden wir in 20 bis 30 Jahren einen Totalschaden. Eine Studie hat eben ergeben, dass die Zahl jener, die über 85 Jahre alt sind und in die Notfall-Station des Universitätsspitals CHUV in Lausanne eingeliefert werden, zwischen 2005 und 2010 um rund 50 Prozent zugenommen hat» [1]. Schon heute beschäftigt sich die Familienmedizin zum grössten Teil mit Senioren - das Modell des Hausarztes, der sich um die Kinder und die Grosseltern gleichermassen kümmert, der sowohl Entbindungen als auch chirurgische Eingriffe durchführt, ist ausgestorben.

Um zu untersuchen, welche Massnahmen die richtigen sind, sind deskriptive Daten wichtig. In dieser Hinsicht sind Kohortenstudien sehr nützlich. Wir erinnern uns an die Vorgänger; die Framingham Heart Study, die 1948 bei Boston begonnen wurde, die Whitehall Study an britischen Beamten ab 1967 sowie mehrere Kohorten von Menschen mit HIV, unter anderem in der Schweiz. Was die Erforschung des Alterns angeht, hat «Lausanne cohorte 65+» (Lc65+) kürzlich ihr 10-jähriges Bestehen gefeiert [2]. Hierbei handelt es sich um eine Forschungsplattform, die zwei Ziele verfolgt: den Bedarf besser zu verstehen, um das Gesundheitssystem anzupassen, und den Abbau der körperlichen Verfassung im Alter zu untersuchen. Dem letzten Bulletin der Initiative ist zu entnehmen, dass die Teilnehmer, die 2004 zwischen 65 und 70 Jahren alt waren, ihren Gesundheitszustand acht Jahre später noch zu zwei Dritteln als gut oder sehr gut beschreiben. An erster Stelle der Gesundheitsbeschwerden stehen bei ihnen Bluthochdruck und Arthrose; und die Faktoren, die ihnen die grössten Sorgen bereiten, sind die Sicherheit auf der Strasse und die finanziellen Ressourcen. Bei etwas mehr als einem Viertel wurde der Zustand als Vorstufe der Gebrechlichkeit eingestuft. Der «Phänotyp Gebrechlichkeit» ist deutlich ausgeprägter bei denjenigen, die unter ungünstigen wirtschaftlichen Bedingungen leben (was eine Konstante der Public Health bestätigt). Im Hinblick auf einen eventuellen Aufenthalt im Heim ist der grösste Wunsch der Befragten ein eigenes Zimmer mit Bad.

An dieser Stelle eine «anthropologische Zwischenbemerkung»: Man spricht viel von der Achtung und der Autorität, die ältere Menschen in traditionellen Gesellschaften beispielsweise in Afrika geniessen, und die davon zeugen, dass die Alten noch ein wichtiger Teil der Gesellschaft sind. Dass diese «Ehrfurcht» in industrialisierten Gesellschaften zurückgeht, hat mehrere Gründe. Zum einen ist es nichts Besonderes mehr, ein hohes Alter zu erreichen - man gilt nicht mehr als «von den Göttern gesegnet». Zum anderen sind die Alten insofern keine Autoritäten mehr, als ihre technischen Kompetenzen durch den digitalen Wandel mit seinem enormen Potential überholt sind.

Manche scheinen bereit, den seit den 1950er Jahren geltenden Generationenvertrag in Frage zu stellen. Jeder sieht die Gefahren für die Systeme, die der Sozialund Solidarstaat für die Senioren errungen hat (zuallererst die AHV, aber auch das Netzwerk für häusliche Pflege Spitex und die APH ...), und jeder ist sich bewusst, dass jüngere Generationen möglicherweise keine Versorgung auf diesem Niveau mehr geniessen können. Zusätzlich vergrössert wird die Gefahr einer «Spaltung» durch die zunehmende Ungleichverteilung der Ressourcen (absolut und/oder relativ betrachtet), die in der ganzen Welt zu beobachten ist - sowohl von Land zu Land als auch innerhalb der Länder.

Nehmen wir diese Entwicklungen ernst genug? Ich würde nicht darauf wetten (nicht zuletzt, weil niemand schlechte Nachrichten mag). Doch einige bemühen sich darum [3]. S. Monod: «Immer muss uns die Frage interessieren, ob eine Massnahme oder eine Technologie gerechtfertigt ist, weil damit die Lebensqualität eines Patienten verbessert werden kann. Neuro-Roboterchirurgie oder Protonen-Therapien braucht es auch, im Vordergrund aber stehen für mich die Hunderte, für die wir keinen Platz, kein Bett in einer geeigneten Institution finden.» Ich wünsche unserer Kollegin, der Fachärztin für Geriatrie, die nun Chefin, Managerin, Planerin, Verhandlungsführerin/Diplomatin, gesellschaftliche Akteurin wird, von Herzen das Beste für ihre neuen Aufgaben. Bernanos: «Die Zukunft ist nicht einfach hinzunehmen, sondern zu gestalten» [4].

Jean Martin, Mitglied der Redaktion 